\title{
Deterministic Component of 2-D Wold Decomposition for Geometry and Texture Descriptors Discovery
}

\author{
Erika Danaé López-Espinoza and Leopoldo Altamirano-Robles \\ National Institute of Astrophysics, Optics and Electronics, \\ Computer Science Department, \\ Luis Enrique Erro 1, 72840 Tonantzintla, México \\ $\{$ danae, robles $\}$ @inaoep.mx \\ http://ccc.inaoep.mx
}

\begin{abstract}
In this paper, the deterministic component of 2-D Wold decomposition is used to obtain texture descriptors in industrial plastic quality images, and hidden geometry of tree crown in remote sensing images. The texture image is decomposed into two texture images: a non-deterministic texture and a deterministic one. In order to obtain texture descriptors, a set of discriminant texture features is selected from the deterministic component. The texture descriptors have been used to distinguish among three kinds of plastic quality. The obtained texture descriptors are compared against texture descriptors obtained from the original image. With the objective to find hidden geometry of tree crown in remote sensing images, the deterministic component of the original image is analyzed. The observed geometry is compared against the modeled geometry in the literature of marked point processes.
\end{abstract}

Keywords: 2-D Wold Decomposition, Homogeneous Random Fields, Texture, Geometry.

\section{Introduction}

Texture analysis methods have been traditionally divided into two categories [10]. The first one, called the statistical or stochastic approach, treats textures as statistical phenomena. A textured pattern is characterized by either statistical of image pixel gray values or some stochastic model. The stochastic formulation of a texture is based on a model in which a texture is viewed as a sample of a 2-D stochastic process describable by its statistical parameters. The second category, called the structural approach, introduces the concept of texture primitives, often called textel or textons (texture element). In order to describe a texture, a vocabulary of textels and placement rules that define the exact location of each primitive in the texture are needed. The goal is to describe complex structures with simpler primitives. However, most natural textures do not fall into any of these two categories and it is difficult to classify textures by one single method. Therefore, unified models have been proposed, in which the deterministic and 
non-deterministic component of a texture field is separated. The 2-D Wold-like decomposition accomplishes this task. This unified texture model proposed by Francos et al. 3. provides a means to analyze the stochastic and deterministic part of a texture separately. The 2-D Wold decomposition has been applied to the image retrieval [6] and for estimating and coding of texture [8]. In [5] a Hough transformation is used to detect spectral evanescent components whereas in [9] a new estimation algorithm of the evanescent field is given. This new method is based on a projection approach and requires a set of projection directions which is obtained by using Farey's series. Now current work propose a new segmentation algorithm which takes into account the deterministic component of 2-D Wold decomposition, as the image of the external field in the MRF model 4. Moreover, in 11 are explored the properties of higher order statistics and Wold decomposition theory for the sake of finding an algorithm for 3-D texture decomposition.

In the proposed model by Francos, the texture field is assumed to be a realization of a 2-D homogeneous random field. Based on a 2-D Wold-like decomposition of homogeneous random field, the texture field is decomposed into a sum of two mutually orthogonal components: a structural or deterministic component, which results in the structural attributes of the observed realization, and a purely stochastic or purely non-deterministic component, which represents the randomness of the texture field. The deterministic component is further decomposed into a sum of two orthogonal components: a harmonic component and a generalized evanescent component. The harmonic component represents the periodic attributes of the texture, whereas the evanescent components represent directional ones. Then, the perceptual property of texture images, randomness, directional, and periodical are preserved (see Fig. 11).

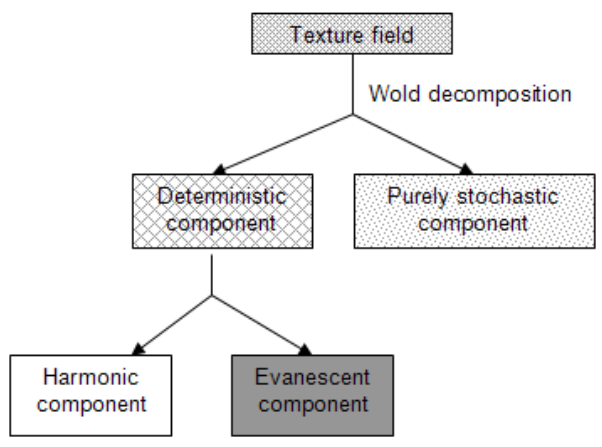

Fig. 1. Wold decomposition

Texture discrimination plays a vital role in real world image segmentation and object extraction. In order to obtain this discrimination, texture exact features have to be extracted. A good texture feature must determinate both similarities into classes and dissimilarities inter classes. In industrial applications is 
frequently to classify similar textures belonging to different classes, therefore, is necessary to consider helpful texture descriptors in these classification problems.

Automatic extraction of objects from several remotely sensing images (such as aerial and satellite images) is a very important research task in pattern recognition and image understanding. Many objects in remote sensing have often some regular shape attributes, such circle, ellipse, polygon etc. and they are commonly distributed orderly and aggregated into different object groups. The automatic extraction of tree crown from remotely sensed images by means of marked point processes is reported in [7]. Perrin et al. model the stand as a realization of a marked point processes of ellipses or ellipsoids, whose point are the positions of the trees and the marks their geometric features. Moreover, there are other vegetation natural resources important to study in the scale of trees whose geometry is unknown e.g. mangrove.

In this paper, we are motived by Franco's model in which a texture is decomposed into constituent components allowing an analysis of the individual components, the deterministic component is used to obtain texture descriptors of three kinds of plastic quality and to find hidden geometry of tree crown in remote sensing images. The implemented algorithm is based on the harmonic field process of [3] and the evanescent field process of [5]. The obtained texture descriptors are compared against texture descriptors obtained from the original image and the observed geometry is compared against the modeled geometry in the literature of marked point processes.

\section{2-D Wold Decomposition of Homogeneous Random Fields}

Consider a homogeneous and regular random field $y(m, n),(m, n) \in Z^{2}$. The 2-D Wold decomposition allows the field to be decomposed in two mutually orthogonal components [3]:

$$
y(n, m)=v(n, m)+w(n, m)
$$

where $\{v(n, m)\}$ is deterministic and $\{w(n, m)\}$ is non-deterministic. The deterministic component can be decomposed in the harmonic component $\{h(n, m)\}$ and evanescent component $\{g(n, m)\}$ :

$$
v(n, m)=h(n, m)+g(n, m)
$$

In the frequency domain, the spectral distribution fuction (SDF) of $\{y(n, m)\}$ can be uniquely represented by the SDF's of its component fields:

$$
F_{y}(\omega, v)=F_{v}(\omega, v)+F_{w}(\omega, v)
$$

where $F_{v}(\omega, v)=F_{h}(\omega, v)+F_{g}(\omega, v)$, and the functions $F_{h}(\omega, v)$ and $F_{g}(\omega, v)$ correspond to spectral singularities supported by point-like and line-like regions, 
respectively. Under some assumptions and approximations Francos et al. [3] proposed the following models:

The harmonic random field has the following representation:

$$
h(n, m)=\sum_{k=1}^{P}\left\{C_{k} \cos 2 \pi\left(n \omega_{k}+m v_{k}\right)+D_{k} \sin 2 \pi\left(n \omega_{k}+m v_{k}\right)\right\}
$$

where the $C_{k}$ 's and $D_{k}$ 's are mutually orthogonal random variables, and $\left(\omega_{p}, v_{p}\right)$ are the spatial frequencies of the $k$ th harmonic. This component generates the 2D delta functions of the "spectral density" (The 2-D delta functions are singular functions supported on discrete points in the frequency plane).

A model for the evanescent field $\{g(n, m)\}$, which corresponds to the RNSHP (rational non-symmetrical half-plane) defined by $(\alpha, \beta) \in O$ is given by a linear combination of fields $e(n, m)$ of the form:

$$
e(n, m)=s(n) \sum_{i=1}^{I}\left\{A_{i} \cos 2 \pi m v_{i}+B_{i} \sin 2 \pi m v_{i}\right\}
$$

where $\{s(n)\}$ is a purely non-deterministic 1-D process, $I$ is the number of evanescent components with $(\alpha, \beta)$ slope and $v_{i}$ is the frequency of the $i$ th evanescent component.

The purely non-deterministic component is given by

$$
w(n, m)=-\sum_{(0,0) \prec(k, l)} b(k, l) w(n-k, m-l)+u(n, m)
$$

where $\{u(n, m)\}$ is the 2-D white innovations field, whose variance is $\sigma^{2}$.

Further details of the Eq. 4, 5] and [6] can be found in [3].

\section{The Texture Model - Implementation}

In general, the analysis algorithm of Francos is based on three stages:

- First, the parameters of the harmonic field are estimated (periodic features).

- Second, the evanescent components of the texture field are estimated (global directional features).

- Finally, the parameters of the purely non-deterministic component of the texture field are estimated (random features).

The texture reconstruction is carried out by summing the images obtained by the inverse procedure to the analysis procedure. Further details of the analysis algorithm can be found in 3 . We only analyze the deterministic component of the analysis algorithm, because this component is parameterized independently of the purely non-deterministic component. The deterministic component is obtained through a global thresholding procedure, proposed in [3], which estimate the harmonic texture field, and a Hough transformation procedure proposed in [5], that estimate the evanescent components. The algorithm to obtain the deterministic component was developed in MATLAB 7.1 and the implementation details are shown in the following sections. 


\subsection{Harmonic Peak Detection}

First, we search the presence of the harmonic components before estimating their parameters. The harmonic peaks are detected in the Fourier magnitude image. The periodogram is a frequency estimator for the unknown periodic components by choosing the frequencies of the largest and sharpest isolated peaks of the periodogram. The amplitude threshold value is established to the maximal value of the periodogram. A magnitude histogram is built over the Fourier magnitude image to establish the minimum value or cut frequency. The number of detected spectral peaks are the harmonic component and are denoted by $P$, see Eq. 4 . The parameterization is done by evaluating the amplitude and the phase values of the texture DFT at the frequencies of the detected maxima. The process to estimate harmonic peaks is shown in Fig. 2.

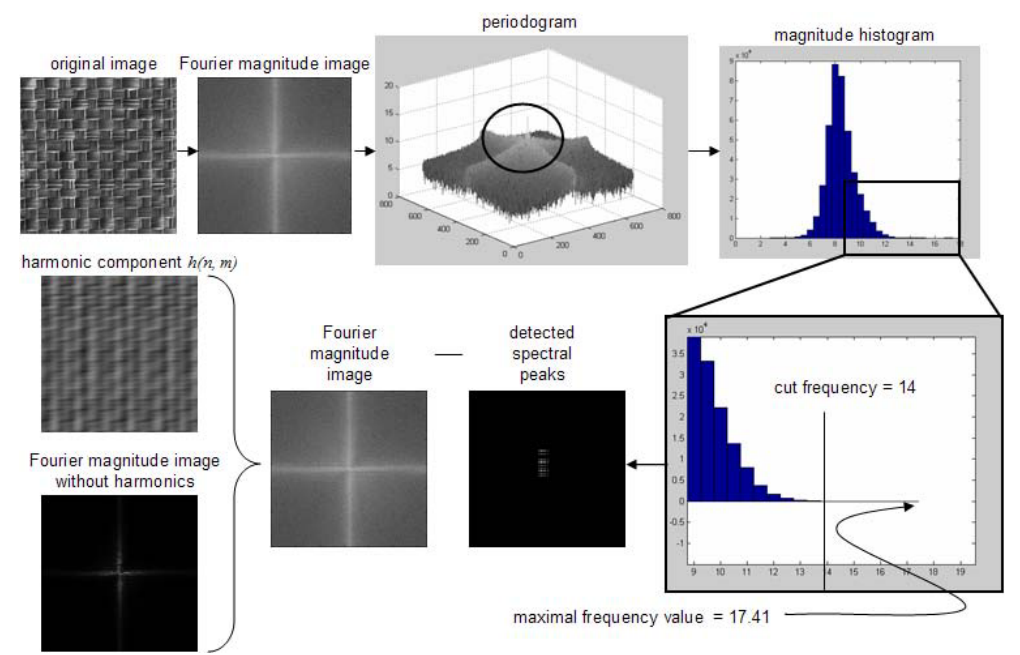

Fig. 2. Process to estimate the harmonic component. Brodatz texture D64 [1].

\subsection{Evanescent Line Detection}

In this case we search for the frequencies of the periodogram largest peaks such that the peaks are located in neighboring frequencies along one dimension. The Hough transformation method for line detection is used to detect the evanescent lines in the Fourier magnitude image. Prior to applying the Hough transform, the spectral values associated with the harmonic peaks should be removed. Both amplitude and phase components of the DFT are retained to parameterization. The process to estimate evanescent lines is shown in Fig. 3, where six lines are found for the Brodatz texture D64.

Finally, the deterministic component (see Fig 4) is estimated by summing the images obtained by the inverse procedure to the harmonic peaks and evanescent lines detection. 


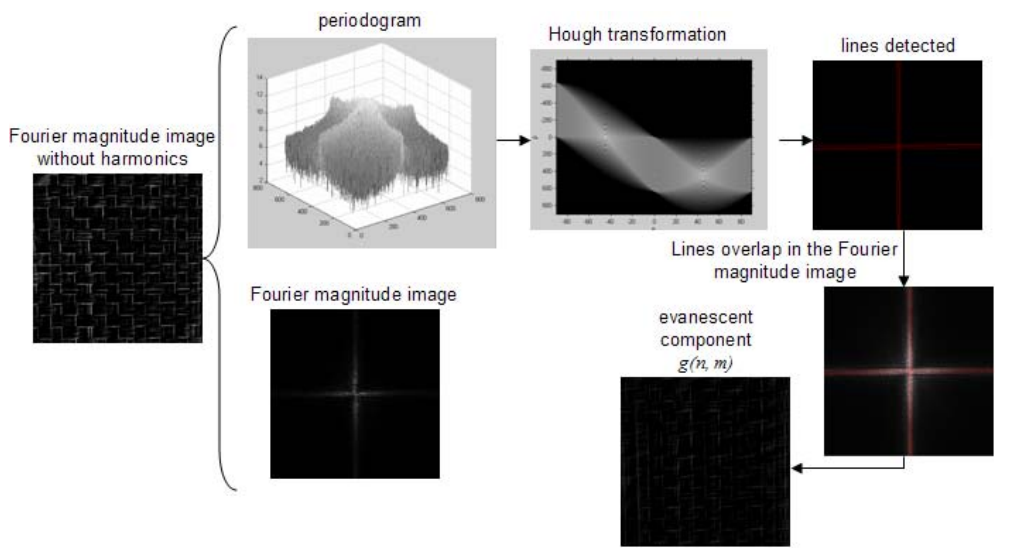

Fig. 3. Process to estimate the evanescent lines

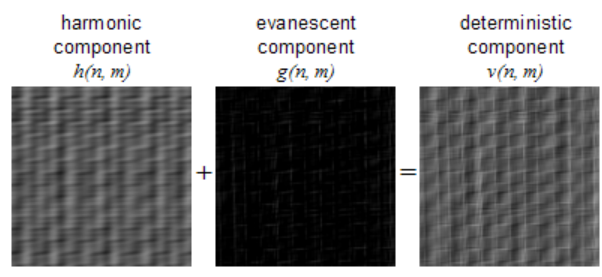

Fig. 4. Structural component of Brodatz texture D64

\section{Experimental Results}

The experimental results are presented in two parts: first, the deterministic component of Wold decomposition is used to obtain descriptors that discriminate among three classes of plastic quality. Second, the deterministic component is used to extract hidden geometry of trees in texture images.

\subsection{Texture Descriptors}

The plastic database includes 52 images of plastic quality labeled manually. The images were obtained directly from the industry. The classification error in this manually labeled data is around $40 \%$. The three plastic quality classes are shown in Fig. 5. It can be seen that is difficult to distinguish among the three plastic quality classes because they present very similar characteristics. The database has 15 samples of class 1,18 samples of class 2 and 19 samples of class 3 . The size of the images is $640 \times 640$ pixels. For the experiments the images were converted to gray scale.

In this experiment, four texture characteristics were obtained for each image: energy, correlation, homogeneity and contrast. Each characteristic was obtained 


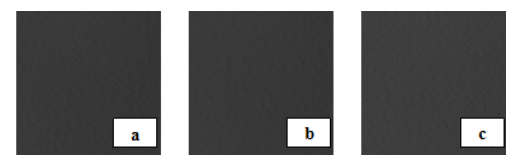

Fig. 5. Images of three plastic quality classes. a)class $1(3)$, b)class 2 (4-) and c)class $3(4+)$.

in four directions $\left(0^{\circ}, 45^{\circ}, 90^{\circ}\right.$ and $\left.135^{\circ}\right)$. Because manual classification presents high ambiguity, we follow an unsupervised approach. The four characteristics were used as texture descriptors to classify with k-means. The texture characteristics (texture descriptors) were obtained for the original images and the deterministic components. The texture descriptors obtained from deterministic component image (TD-DCI) are compared visually against texture descriptors obtained from original image (TD-OI). The obtained clusters using TD-OI and TD-DCI, are shown in Fig. 6. Graphics 6a, 6r, 6e and 6r show clustering of TD-OI in the directions $0^{\circ}, 45^{\circ}, 90^{\circ}$ and $135^{\circ}$ respectively. Graphics 6 $\mathrm{b}, 6 \mathrm{~d}, 6 \mathrm{f}$ and $6 \mathrm{~h}$ show clustering using TD-DCI in the same directions. In deterministic component, the cut frequency determined for the harmonic component is 9.25 of amplitude, and two evanescent lines for each image were found. It can be seen in graphics that the clusters using TD-DCI are better grouped than TD-OI. Using TD-OI the cluster aren't formed. In the experiments, the classification rate using $\mathrm{k}$-means with TD-DCI is $10 \%$ greater than k-means with TD-OI.

\subsection{Geometry}

The second experiment was carried out on aerial and satellite images. The size of the images is variable. The objective of this experiment is to relate the modeled geometry in [7] for tree crown detection with the geometry observed from the deterministic component of the Wold decomposition. In a marked point process frequently the tree crown is modeled by means of circles and ellipses. Fig. 7 shows the deterministic component obtained from the aerial image (Fig. 7b, 7f) in [7. The found geometry is represented by circles in the same way as the modeled geometry done by Perrin (Fig. 75, 7b). A filter to improve the contrast of deterministic component was applied. Obtaining the border of the objects or carrying out visual inspections help us to find the geometry, however, in stochastic textures the geometry is not obvious. Fig. $7 \mathrm{~d}$ and $7 \mathrm{~h}$ show the borders obtained from the original image. It can be seen that deterministic component is more similar to modeled geometry than borders. Fig. 8 a and $8 \mathrm{~b}$ show the found ellipses in a CIR (color infrared) image. Fig. 8d and 8f show the circles and triangles geometry found in a high-resolution satellite image.

\section{Conclusions}

In this paper, the deterministic component of 2-D Wold decomposition was used to obtain texture descriptors and geometry of texture fields. The implemented 

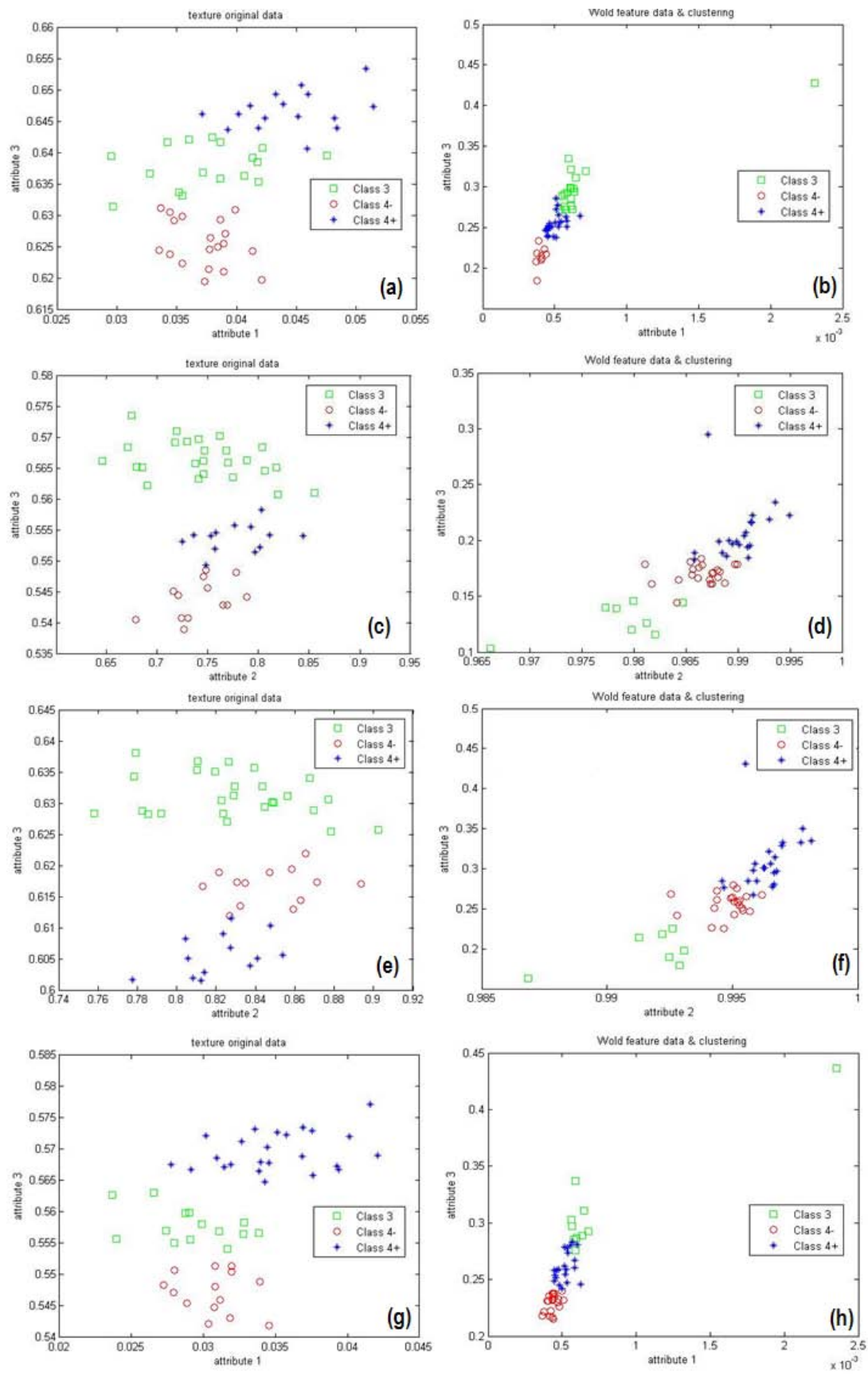

Fig. 6. Comparison of clusters in plastic images (axes $\mathrm{x}$ and $\mathrm{y}$ attributes, $*$ class $4+$, $\circ$ class $4-, \square$ class 3). 6a, 6c, 6e and $6 \mathrm{~g}$ show clusters of TD-OI in $0^{\circ}, 45^{\circ}, 90^{\circ}$ and $135^{\circ}$ respectively; $6 \mathrm{~b}, 6 \mathrm{~d}, 6 \mathrm{f}$ and $6 \mathrm{~h}$ show clusters using TD-DCI in the same directions. 


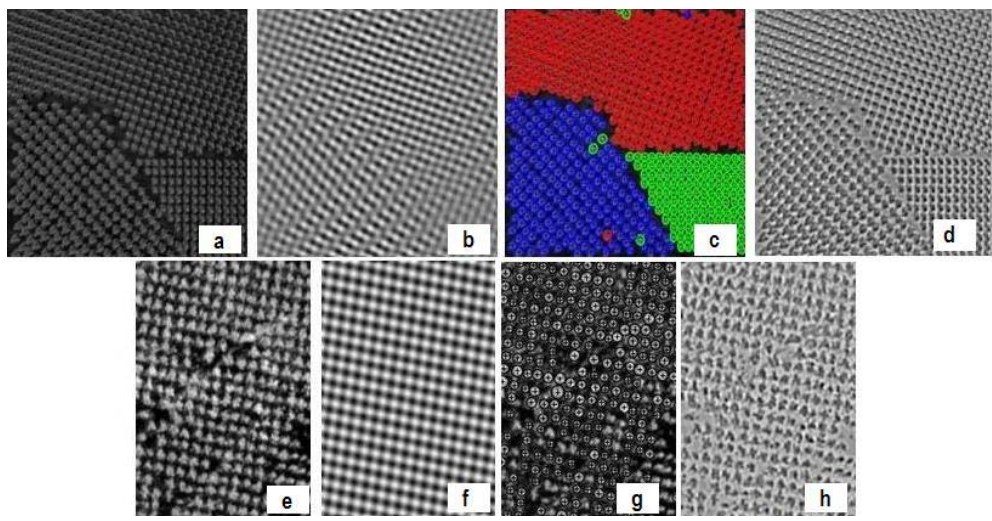

Fig. 7. Geometry: a) and e) original image, b) and f) deterministic component, c) and g) modeled geometry, d) and h) border image

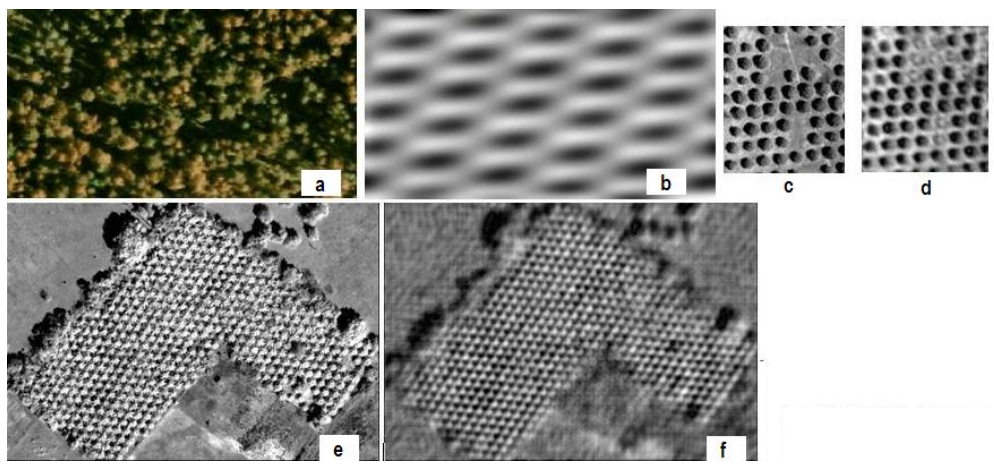

Fig. 8. Geometry for CIR and high-resolution satellite image of permanent cover (agriculture): a), c) and e) original image, b), d) and f) deterministic component

algorithm considers a global thresholding process and other processes based in Hough transformation to estimate the deterministic component. Experimental results show that texture descriptors obtained from deterministic component groups better the classes than texture descriptors from original image. As well, experimental results to find the geometry of tree crown in images show that the obtained geometry from Wold decomposition is the same geometry modeled in marked point processes. Future work involves modeling several covers as a marked point process and defining precisely the geometry obtained from Wold decomposition as the mark of the point process.

Acknowledgments. The first author acknowledges to CONACYT the support provided through the grant for Ph.D. studies number 171464. The first author also acknowledges to Octavio Gómez Ramos for his valuable comments. 


\section{References}

1. Brodatz, P.: Textures: A Photographic Album for Artists and Designers. Dover Publications, New York (1966)

2. Cross, G., Jain, A.: Markov Random Field Texture Models. IEEE Transactions on Pattern Analysis and Machine Intellige 5, 25-39 (1983)

3. Francos, J.M., Meiri, A.Z., Porat, B.: A Unified Texture Model Based on a 2-D Wold-Like Decomposition. IEEE Transactions on Signal Processing 41, 2665-2678 (1993)

4. Li, F., Peng, J., Zheng, X.: Object-Based and Semantic Image Segmentation Using MRF. EURASIP Journal on Applied Signal Processing 6, 840-883 (2004)

5. Liu, F., Picard, R.W.: A Spectral 2-D Wold Decomposition Algorithm for Homogeneous Random Fields. In: IEEE International Conference on Acoustics, Speech, and Signal Processing, vol. 4, pp. 3501-3504. IEEE Signal Processing Society, Piscataway New Jersey (1999)

6. Liu, F., Picard, R.W.: Periodicity, Directionality, and Randomness: Wold Features for Perceptual Pattern Recognition. Computer Vision \& Image. In: Proceedings of the 12th IAPR International Conference on Pattern Recognition, vol. 2, pp. 184-189. IEEE Computer Society, Los Alamitos California (1994)

7. Perrin, G., Descombes, X., Zerubia, J.: 2D and 3D Vegetation Resource Parameters Assesment using Marked Point Processes. In: ICPR 2006, vol. 1, pp. 1-4. IEEE Computer Society, Washington DC USA (2006)

8. Sriram, R., Francos, J.M., Pearlman, W.A.: Texture Coding Using a Wold Decomposition Model. In: Proceedings of the 12th IAPR International Conference on Pattern Recognition. IEEE Transactions on Image Processing, vol. 3, pp. 35-39. IEEE Signal Processing Society, Piscataway New Jersey (1996)

9. Ramananjarasoa, C., Alata, O., Najim, M.: 2-D Wold Decomposition: New Parameter Estimation Approach to Evanescent Field Spectral Supports. In: EUSIPCO, vol. 2, pp. 913-916 (2000)

10. Huang, Y., Chan, K.L.: Texture Decomposition by Harmonics Extraction From Higher Order Statistics. IEEE Transactions on Image Processing 13, 1-14 (2004)

11. Stitou, Y., Turcu, F., Najim, M., Redouane, L.: 3-D Texture Characterization Based on Wold Decomposition and Higher Order Statistics. In: ICASSP, vol. 2, pp. 165-168. IEEE Signal Processing Society, Piscataway New Jersey (2005) 